\section{Association between HLA-DR13 and Susceptibility to Alveolar Echinococcosis}

Colleagues-Alveolar echinococcosis (AE), caused by infection with the larval stage of the small fox tapeworm Echinococcus multilocularis, is one of the most lethal helminthic infections in humans. Its pathology and high mortality rate $(56 \%-94 \%$ in untreated cases; $10 \%-14 \%$ after treatment and early diagnosis [1]) are reasons for public health concern.

Prevalence among European fox populations is 35\% in Switzerland [2], which is similar to rates reported for some endemic areas of France and Germany. The wide distribution of foxes infected with egg-producing $E$. multilocularis may represent a considerable potential risk for humans in densely populated regions, such as Switzerland; however, despite this high prevalence in the definitive host, the disease in humans is relatively rare. In Switzerland, the annual morbidity rate has been relatively stable at $0.18 \mathrm{AE}$ cases per 100,000 inhabitants for several decades [1]. Similar data were reported for France, Germany, and Austria [3].

Previous seroepidemiologic studies detected cases of early active, but asymptomatic, $\mathrm{AE}$ and provided the first evidence of self-healed cases of $\mathrm{AE}$ in humans and the presence of inactive dead or aborted larval parasites [4], indicating the potential for different courses of AE in humans.

Experimental infections in inbred laboratory mouse strains have shown that susceptibility and resistance to infection with E. multilocularis metacestode and subsequent disease may be geneticaliy controlled $[5,6]$. Our efforts to understand the mechanisms and genetics of susceptibility to AE have focused on the identification of DNA markers that segregate the disease by populations living in endemic areas. We addressed this task by determining the frequency of the lymphoid cell surface HLA-DR proteins, which are encoded in the region of the major histocompatibility complex and referred to as class II antigens.

Blood samples were collected over 3 years from 32 patients with hepatic AE. The patients were preselected for the study by their homogeneous geographic and ethnic origin (all Swiss) and by their clinical courses (described in detail in [7]). Clinical courses included radical resection of the hepatic metacestode lesion without recurrence; partial resection of the hepatic lesion, continuous mebendazole or albendazole treatment, and regressive or stationary course of disease; inoperable lesion treated with continuous mebendazole or albendazole, and stationary or progressive development of disease. Although the clinical courses of disease differed, the groups matched each other in parameters, such as average age, sex, and disease duration. HLA-DR antigens were determined by oligotyping [8]: Leukocyte DNA had DRB genes amplified by the polymerase chain reaction. This was followed by sequence-specific hybridization with biotinylated oligonucleotides detected in a chemolumines-

Reprints or correspondence: Dr. Bruno Gottstein, Institute of Parasitology. Faculty of Veterinary Medicine. University of Berne. Länggass-Strasse 122, P.O. Box 8466. CH-3001 Berne, Switzerland.

The Journal of Infectious Diseases 1994;169:1416-7 (C) 1994 by The University of Chicago. All rights reserved. $0022-1899 / 94 / 6906-0049 \$ 01.00$
Table 1. Distribution of relevant HLA antigens (typed by polymerase chain reaction sequence-specific oligonucleotides [PCRSSO]) in Swiss patients with alveolar echinococcosis and in healthy Caucasian controls.

\begin{tabular}{|c|c|c|c|c|}
\hline \multirow[b]{2}{*}{$\begin{array}{l}\text { HLA, PCR-SSO-determined } \\
\text { genes }\end{array}$} & \multicolumn{2}{|c|}{$\begin{array}{l}\text { \% phenotype } \\
\text { frequency }\end{array}$} & \multirow[b]{2}{*}{$x^{2}$} & \multirow[b]{2}{*}{$P$} \\
\hline & $\begin{array}{l}\text { Patients } \\
(n=32)\end{array}$ & $\begin{array}{c}\text { Controls } \\
(n=177)^{*}\end{array}$ & & \\
\hline DRI, DRB $1 * 0101-0103$ & 12.5 & 26.0 & & \\
\hline DR2, DRB5*0101, 0102, 0201, 0202 & 37.5 & 31.6 & & \\
\hline DR3, DRB 1*0301. 0302 & 15.6 & 23.7 & & \\
\hline DR4, DRB 1*0401-0411 & 15.6 & 41.3 & 6.5 .0 & 01 \\
\hline DR7. DRB $1 * 0701,0702$ & 31.3 & 25.4 & & \\
\hline DR8, DRB I*0801-0804 & 9.4 & 4.6 & & \\
\hline DR9. DRBI*090I & 3.1 & 2.8 & & \\
\hline DR 10, DRB I*1001 & 0 & 0.6 & & \\
\hline DRII, DRBI*1101-1104 & 12.5 & 11.3 & & \\
\hline DR 12, DRB $1 * 1201,1202$ & 3.1 & 2.8 & & \\
\hline DR13. DRB 1*1301, I 302 & 34.4 & 13.6 & 7.0 & .008 \\
\hline DRB $1^{*} 1303$ & 0.6 & 5.1 & & \\
\hline DRB $\left.\right|^{*} 1304$ & 0 & 0.6 & & \\
\hline DR 14, DRB $1 * 1401,1404$ & 3.1 & 5.6 & & \\
\hline DRx, Not detected or homozygotic & 18.8 & 10.6 & & \\
\hline
\end{tabular}

${ }^{*}$ DR distribution determined by PCR-SSO [10]

cent assay [9]. The relevant distribution of HLA-DR antigens in patients with AE versus a random Caucasian control group is shown in table 1.

In our patient group, the frequency of HLA-DR 13 (DRBI*1301/1302) antigen was significantly increased (patients, $34.4 \%$; controls, $13.6 \% ; \chi^{2}=7.0, P=.008$ ). The association between an HLA antigen and susceptibility to disease after infection with $E$. multilocularis was highly significant. Subgrouping of patients with respect to individual clinical status and course provided more insight into the distribution of the respective DR 13. In the group of inoperable patients, 2 (12\%) of 14 expressed DR 13 as did 4 (40\%) of 10 of those who had partial surgery and $6(75 \%)$ of 8 who had radical resection. Our primary finding was that persons expressing DR 13 develop AE with a much higher frequency (and a relative risk factor of 5.7) than will persons not expressing DR 13. By subgrouping patients by clinical status and course, it was obvious that once infection resulted in disease, a DR 13 predisposition was favorable and radical surgery was much more likely. Why this is so remains unknown. We postulate that in patients with DR13, due to higher susceptibility to disease, symptoms may become manifest at an earlier stage of infection when radical surgical resection is more likely.

The present study also shows a reduced frequency of HLADR4 antigens among patients versus controls (patients, 15.6\%; controls, $41.3 \% ; \chi^{2}=6.5 ; P=.01$; protection factor, 0.6 ). Although not highly significant, this finding and knowing from previous epidemiologic studies that a certain portion of persons infected with $E$. multilocularis will not develop disease [1], or will cast off organisms spontaneously at an early stage [4], provides the rationale for further HLA-DR studies in families of patients at high risk of infection, such as native Yupik popula- 
tions of Alaska, and for reversed elucidation of potential genetic resistance markers indicating metacestode abortion [4].

\section{Bruno Gottstein and Florence Bettens}

Institute of Parasitology. University of Berne, and Institute of Clinical Immunology. Inselspital, Berne, Switzerland

\section{References}

1. Gottstein B. Echinococcus multilocularis infection: immunology and immunodiagnosis. Adv Parasitol 1992;31:321-80.

2. Ewald D, Eckert J, Gottstein B, Straub M, Nigg H. Parasitological and serological studies on the prevalence of Echinococcus multilocularis Leuckart. 1863 in red foxes (Vulpes vulpes Linnaeus, 1758) in Switzerland. Rev Sci Tech 1992;11:1057-61.

3. World Health Organization. Report of the WHO informal consultation on Echinococcus mulitocularis research. Geneva: WHO 1988.

4. Rausch RL, Wilson JF, Schantz PM, McMahon BJ. Spontaneous death of Echinococcus multilocularis: cases diagnosed serologically by Em2ELISA and clinical significance. Am J Trop Med Hyg 1987;36:57685.

\section{Nitric Oxide in Cerebral Malaria}

Colleagues-Cerebral malaria is an important complication of Plasmodium falciparum infection and a major cause of infant mortality in Africa. The pathophysiology of cerebral malaria remains incompletely understood. Recently, a link between cytokines, nitric oxide (NO), and cerebral malaria was proposed $[1,2]$. It was hypothesized that intravascular NO, induced by tumor necrosis factor in endothelial cells and vascular smooth muscle, diffuses through the blood-brain barrier and interferes with neurologic function. Furthermore, NO is thought to increase intracranial pressure, a common feature in cerebral malaria in African children [3], through increased vasodilatation. Our data do not support this concept.

We studied the evolution of NO plasma concentrations in 28 African children (mean age $\pm \mathrm{SD}, 4.4 \pm 3$ years) with cerebral malaria in Yaoundé. All children presented with unrousable coma and $P$. falciparum parasitemia. Coma depth was assessed according to the modified Glasgow coma scale for young children [4]. Other causes of coma were excluded. After treatment with quinine ( $25 \mathrm{mg}$ of base $/ \mathrm{kg}$ daily for 8 days), the clinical outcome was favorable in 21 cases; 4 children developed neurologic sequelae. Three children died: after $12 \mathrm{~h}, 3$ days, and 4 days. Mean duration of coma for the 25 surviving children was $45.1 \pm 31.4 \mathrm{~h}$. NO plasma concentrations were measured on the day of admission (day 1) and on days 2, 3, 5, and 8 (discharge). Stable $\mathrm{NO}$ end products $\left(\mathrm{NO}_{2}^{-}\right.$and $\mathrm{NO}_{3}^{-}$) were measured by means of an automated procedure, as described previously [5].

On day 1 , mean NO concentration in 28 children was $44.3 \pm$

Reprints or correspondence: Dr. Pascal Ringwald, ORSTOM/OCEAC, BP288, Yaoundé, Cameroon.

The Journal of Infections Diseases 1994;169:1417-8

(C) 1994 by The University of Chicago. All rights reserved.

$0022-1899 / 94 / 6906-0050 \$ 01.00$
5. Liance $M$, Vuitton D, Guerret-Stocker S, Carbillet JP, Grimaud JA, Houin R. Experimental alveolar echinococcosis. Suitability of a murine model of intrahepatic infection by Echinococcus Mullilocularis for immunological studies. Experientia 1984;40:1436-9.

6. Kroeze WK. Tanner CE. Echinococcus multilocularis: susceptibility and responses to infection in inbred mice. Int J Parasitol 1987;17:87383.

7. Gottstein B, Mesarina B, Tanner I, et al. Specific cellular and humoral immune responses in patients with different long-term courses of alveolar echinococcosis (infection with Echinococcus multilocularis). Am J Trop Med Hyg 1991;45:734-42.

8. Tiercy JM, Jeannet M, Mach B. A new approach for the analysis of HLA class II polymorphism: HLA oligotyping. Blood Rev 1990;4:915.

9. Bettens F, Pichler WJ, de Weck Al.. Incorporation of biotinylated nucleotides for the quantification of PCR-amplified HIV-1 DNA by chemiluminescence. Eur J Chem Clin Biochem 1991;29:685-8.

10. Doherty DG, Vaughan RW, Donaldson PT, Mowatt AP. HLA DQA. DQB and DRB genotyping by oligonucleotide analysis: distribution of alleles and haplotypes in British caucasoids. Hum Immunol 1992;34:53-63.

$36.5 \mu M$. Children with favorable outcome presented with a higher mean NO concentration $(49.9 \pm 40.3 \mu M)$ than those with neurologic sequelae $(30.6 \pm 15.5 \mu M)$ or those who died $(23.7 \pm 4.6 \mu M)$. NO concentrations were higher in children with a coma score of $3(n=11 ; 63.2 \pm 49 \mu M)$ than in children with a coma score of 1 or $2(n=17 ; 32.2 \pm 18.6 \mu M)$. These differences were not statistically significant. NO concentrations were negatively correlated with the duration of coma (Spearman rank test, $r^{\prime}=-.49, P<.02$ ), indicating that the duration of coma was significantly longer in children with NO plasma levels $<44.3 \mu M$ than in those with NO plasma levels superior to the mean $(53.6 \pm 27.5$ vs. $32.4 \pm 34.0 \mathrm{~h} ; P<.02)$. No significant correlation between NO level and platelet or white blood cell counts, blood glucose level, parasitemia, hemoglobin level, or body temperature was found.

We observed an increase of mean NO concentration after 24 $h$ of quinine treatment in children with favorable outcome (group A); in children with sequelae (group B), this was found after $48 \mathrm{~h}$. By day 3, mean NO level was higher in group $A$ ( 44.2 $\pm 50.9 \mu M)$ than in group B $(12.8 \pm 3.9 \mu M)$ (Mann-Whitney $U$ test. $P=.03)$. Similar results were observed at day $5(62.1 \pm$ 68.1 vs. $16.2 \pm 7.1 \mu M ; P<.04$; figure 1 ).

Although NO plasma concentrations are higher during cerebral malaria than during uncomplicated malaria attacks (unpublished data), our results seem to refute the hypothesis that increased NO levels are detrimental in the development of cerebral malaria. In a murine model, $N$-monomethyl-L-arginine, an inhibitor of NO synthase, prevented neither the development of neurologic symptoms nor the death of Plasmodium bergheiinfected mice $[6,7]$. Furthermore, treatment with a competitive NO synthase inhibitor had a deleterious effect in complicated murine Plasmodium vinckei malaria [8]. Therefore, it has been suggested that NO has a protective role in cerebral malaria [9]. Further investigations are being done to confirm the protective effect of NO in human pathology as suggested by murine models and by our preliminary results. 